\title{
Power Allocation Strategy in NOMA-Based Cloud Radio Access Networks
}

\author{
Wei Xu \\ Key Laboratory of Universal Wireless \\ Communications, Ministry of Education, Beijing \\ University of Posts \& Telecommunications \\ Beijing, China 100876 \\ vivi439@bupt.edu.cn
}

\author{
Yong Li \\ Key Laboratory of Universal Wireless \\ Communications, Ministry of Education, Beijing \\ University of Posts \& Telecommunications \\ Beijing, China 100876 \\ liyong@bupt.edu.cn
}

\begin{abstract}
This paper investigates power allocation strategy for a small cell in cloud radio access networks (C-RANs) applying non-orthogonal multiple access (NOMA) technique in an ideal scenario without inter-cell interference and fronthaul constraint. We firstly formulate an optimization problem in terms of decoding order and power allocation among multiple users to maximize the total throughput under a sum power constraint and quality of service conditions. Then we verify the relationship between channel gain order and decoding order, after that we remove one constraint to relax the problem, and propose a power allocation algorithm with low complexity. Monte Carlo simulation results show that the proposed algorithm outperforms other power allocation strategies.
\end{abstract}

\section{KEYWORDS}

Non-orthogonal multiple access, cloud radio access networks, decoding order, multi-user power allocation

\section{ACM Reference format:}

Wei Xu and Yong Li. 2017. Power Allocation Strategy in NOMABased Cloud Radio Access Networks. In Proceedings of ACM Mobimedia conference, Chongqing, China, July 2017 (MOBIMEDIA'17), 6 pages.

DOI: $10.1145 /$ nnnnnnn.nnnnnnn

\section{INTRODUCTION}

In the coming decade, cellular networks are faced with thousand-fold growing capacity in the mobile data traffic [1][2]. Recently, non-orthogonal multiple access (NOMA) has been regarded as a promising multi-user access technique to be applied in the next generation wireless communication systems for 2020 [3][4], which emphasizes higher quality of service as well as accommodating more users. When NOMA is applied in downlink transmission, users can receive data Permission to make digital or hard copies of all or part of this work for personal or classroom use is granted without fee provided that copies are not made or distributed for profit or commercial advantage and that copies bear this notice and the full citation on the first page. To copy otherwise, to republish, to post on servers or to redistribute to lists, requires prior specific permission and/or a fee.

F-RAN 5G 2017, July 13-14

Copyright @ 2017 EAI 978-1-63190-156-0 differentiation of power domain [5]. More specifically, user data are multiplexed with appropriate power allocation in transmitter, and the transmitted signal of the weaker user must be allocated with much more power than that of the stronger user. In addition, successive interference cancellation (SIC) is utilized in order to separate the composite multiuser signal. In contrast to orthogonal multiple access (OMA), NOMA can offer advantages, such as better theoretical capacity region and improved spectral efficiency [6].

So far, intensive research works have investigated downlink NOMA system. The authors in [7] have researched the performance of a multiuser system for outage probability and the average sum throughput in random user deployment. In [8], the authors have presented some system-level and linklevel simulations to verify the improvement in terms of sum throughput of system and individual user's throughput. To employ NOMA technique, eNB needs to carry out a grouping scheme and power allocation strategy in superposed transmission. In [9], considering the sum throughput as well as cell-edge users' throughput, the authors proposed a weighted proportional fairness-based multiuser scheduling scheme. Further, in reality scenario, social relations [10][11] can also be considered for users to form groups. As assigned power varies among users, power allocation plays a key role in system performance. In [12], the authors presented a power allocation schemes for a downlink NOMA system with an eNB and two users under a total power constraint and minimum rate requirements, and their work focused on single input single output (SISO) and multiple input multiple output (MIMO) scenario. System performance considering channel state information (CSI) based power allocation strategy and predefined quality of service (QoS) based power allocation strategy are studied in [13], and a sub-optimal power allocation method with low complexity is presented in [14]. Besides, NOMA can be applied to other systems, such as energy harvesting networks [15] and cloud radio access network (C-RAN) [16] to further improve spectral efficiency .

In this paper, we pay attention to a new scenario combining NOMA with C-RAN to further enhance the performance of C-RAN system. The significant characteristic of C-RAN is that the baseband processing is centralized and shared among sites in a virtualized baseband unit (BBU) pool. Recently, there have been some works investigating C-RAN system. The authors in [17] provided a cluster content caching structure to improve effective capacity. In [18][19], training-based 
channel estimation and cluster formation are studied. In the architecture of C-RAN, there are many small cells comprising a remote radio head $(\mathrm{RRH})$ and several user equipments (UEs). We assume that the coverage zones of neighbouring small cells are not overlapping due to frequency planning so that inter-cell interference doesn't exist. Furthermore, in order to maximize the total throughput of a user group under a total power constraint and satisfy the QoS of all users, we propose a low complexity multi-user power allocation strategy for a small cell of C-RAN applying NOMA, and we verify the relationship between the order of channel gain and optimal decoding order as well.

The remainder of the paper is organized as follows: Section 2 describes the fundamental of downlink system and presents the system model. In Section 3, the sum throughput maximization problem and the proposed strategy are elaborated. Simulation results and related analysis are to be presented in Section 4 to verify the effectiveness of proposed algorithm. Finally, conclusions and some directions for future works are given in Section 5.

\section{SYSTEM MODEL}

Consider a C-RAN system, in which all RRHs connect to the BBU pool via fronthauls, and users are located at different small cells of the system. Each RRH has a unique antenna, which is used to serve all mobile users in its coverage, and also all users have a single antenna configuration. For simplicity, we focus on a specific scene, in which there are only one RRH and several UEs, considering that there is no inter-cell interference due to frequency planning and/or building isolation, and fronthaul is in ideal state. Besides, according to some predefined rules and conditions, all UEs served in a RRH's coverage zone can be divided into several non-overlapping user groups, and in each user group, user data are transmitted over the same resource. Hence, we consider a downlink system with one $\mathrm{RRH}$, active user set $\boldsymbol{M}=\{1,2, \ldots, m\}$, which is divided into $N$ user groups $\boldsymbol{S}=\left\{S_{1}, S_{2}, \ldots, S_{N}\right\}$ basing on predefined grouping principle. Assume that the RRH has the full knowledge of the channel side information (CSI) and different user groups use the nonoverlapping frequency resource assigned by RRH. Moreover, assume the total transmit power of RRH is $P$ and each user group is assigned the same power $P_{t}=P / N$. Due to the power limitation, power allocated to users satisfies $\sum_{j \in S_{n}} p_{j} \leq P_{t}$, in which $p_{j}$ represents the power assigned to user $j$, which is served at the assigned frequency spectrum. The signal transmitted to user $j$ is denoted as $x_{j}$, and $n_{j} \sim C N\left(0, \delta^{2}\right)$ represents the additive white gaussian noise (AWGN) for user $j$, and $\delta^{2}$ is the noise variance. Hence following the principle of NOMA, the superimposed signal received at any user $j$ of user group $S_{n}$ is shown as

$$
y_{j}=h_{j} \sqrt{p_{j}} x_{j}+h_{j} \sum_{i \in\left\{\boldsymbol{S}_{n} / j\right\}} \sqrt{p_{i}} x_{i}+n_{j},
$$

where $x_{j}$ and $x_{i}$ are modulated symbols of user $j$ and other users respectively, and the channel coefficient between RRH and UE $j$ is denoted by $h_{j}$, which can be expressed as an independent complex Gaussian random variable with zeromean and variance $\delta_{h_{n}}^{2}$.

Hence, without SIC, according to Shannon's capacity formula, the rate of user $j$ of user group $S_{n}$ can be given by

$$
R_{j}^{\prime}=\log _{2}\left(1+\frac{p_{j}\left|h_{j}\right|^{2}}{n_{j}+I_{j}^{\prime}}\right)
$$

where $I_{j}^{\prime}=\sum_{i \in\left\{S_{n} / j\right\}} p_{i}\left|h_{j}\right|^{2}$, and it is the interference caused by other UEs received at user $j$, and due to the lack of SIC, it includes all other users' signals superimposed in the transmitted signal. Therefore, we can know that it is essential to eliminate some interference before demodulating user j's signal, so that there is a need to apply SIC technique.

Because of the total power limitation, power allocated to each user satisfies $\sum_{j \in S_{n}} p_{j} \leq P_{t}$. According to Shannon's theorem, the throughput of user $j$ in user group $S_{n}$ is

$$
R_{j}=\log _{2}\left(1+\frac{p_{j}\left|h_{j}\right|^{2}}{n_{j}+I_{j}}\right)
$$

where $I_{j}$ is the interference received at user $j$, and because SIC process is implemented at receiver to reduce the interference derived from other users on the same frequency resource, users whose data are demodulated later can recover and eliminate other users' data first, so it can remove the interference to some extent, and its residual interference caused by other users in the same group whose data will be decoded later than user $j$. Therefore we have

$$
I_{j}=\sum_{i \in\left\{S_{n} \mid o_{i}<o_{j}\right\}} p_{i}\left|h_{j}\right|^{2},
$$

where $o_{i}$ is the order of decoding data of user $i$ in user group $S_{n}$, satisfying $o_{i} \in\left\{1,2, \ldots,\left|S_{n}\right|\right\}$, and the larger the value is, the earlier the user's data are decoded, besides, $\left|S_{n}\right|$ represents the total number of users in group $S_{n}$. Moreover, following the principle of SIC, user whose signal is decoded earlier is supposed to be allocated much more power, so it can be seen that $p_{i}<p_{j}$ when $o_{i}<o_{j}$. Therefore, the signal interference noise ratio (SINR) of user $j$ with $\mathrm{SIC}$ at its receiver can be written as

$$
S I N R_{j}=\frac{p_{j}\left|h_{j}\right|^{2}}{n_{j}+\left|h_{j}\right|^{2} \sum_{i \in\left\{S_{n} \mid o_{i}<o_{j}\right\}} p_{i}} .
$$

Then the sum rate of user $j$ can be given by

$$
R_{j}=\log _{2}\left(1+\frac{p_{j}\left|h_{j}\right|^{2}}{n_{j}+\sum_{i \in\left\{S_{n} \mid o_{i}<o_{j}\right\}} p_{i}\left|h_{j}\right|^{2}}\right) .
$$

In this paper, we focus on the overall sum throughput. Due to different user group using orthogonal resource, the total throughput can be regarded as the sum of $N$ groups, which can be expressed as

$$
R_{\text {total }}=\sum_{n=1}^{N} R_{S_{n}}=\sum_{i=1}^{N} \sum_{j \in S_{n}} \log _{2}\left(1+\frac{p_{j}\left|h_{j}\right|^{2}}{n_{j}+I_{j}}\right) .
$$




\section{PROBLEM FORMULATION AND PROPOSED POWER ALLOCATION ALGORITHM}

\subsection{Problem formulation}

In this section, according to NOMA principle, the demodulation order and power assignment influence the performance. Therefore we formulate the decoding order and power allocation as an optimization problem in NOMA system.

Assume a decoding order, such as $\boldsymbol{o}=\left\{o_{1}, o_{2}, \ldots, o_{\left|S_{n}\right|}\right\}$, in which $i$ is the member of user group $S_{n}$, such that $i \in S_{n}=$ $\left\{1,2, \ldots,\left|S_{n}\right|\right\}$. And if $o_{i}<o_{j}$, then user $j$ 's data are decoded earlier than user $i$. As receiver uses successive interference cancellation, if the corresponding order is larger than the other user's order, receiver will decode the data first and then remove it from the received superposed signal. So that user whose data are decoded before current user will not affect the performance of the receiver.

And there is a need to decide the decoding order and power assignment to maximize the sum utility of members. So this problem can be formulated as an optimization problem, whose optimization variables are each user's power and the decoding order. Besides, due to the fact that all user groups are assigned non-overlapping spectrum resource with the same transmit power $P_{t}$, the optimization problem can be decomposed as $N$ optimization subproblems, in which decoding order and power allocation strategy of a user group are regarded as optimization variables. According to the analysis above, the optimization of total throughput of a arbitrary user group $S_{n}$ is given by

$$
\begin{aligned}
\max _{\boldsymbol{p}, \mathbf{o}} & R_{S_{n}} \\
\text { s.t. } & \mathrm{C} 1: \sum_{j \in S_{n}} p_{j} \leq P_{t}, \\
& \mathrm{C} 2: R_{j} \geq \widetilde{R}_{t h}, \forall j \in S_{n}, \\
& \mathrm{C} 3: R_{i \rightarrow j} \geq \widetilde{R}_{t h}, \forall i \in S_{n} / j, o_{i}>o_{j}, \\
& \mathrm{C} 4: p_{i}>p_{j}, \forall i \in S_{n} / j, o_{i}>o_{j}, \\
& \mathrm{C} 5: p_{j} \geq 0, \forall j \in S_{n} .
\end{aligned}
$$

Constraint $C 1$ represents the power limitation in the downlink transmission, and $P_{t}$ is the power allocated to user group $S_{n}$, and the value of power should be no less than zero. Constraint $C 2$ stresses the fact that after SIC process each user's rate has to be no less than the threshold $\widetilde{R}_{t h}$, otherwise user $j$ can't successfully demodulate its own data. In constraint $C 3$, $R_{i \rightarrow j}$ is user $i$ 's throughput received at user $j$, considering the characteristic of successive interference cancellation (SIC) process, at the receiver user $j$, when its decoding order is later than user $i$, it has to decode user $i$ 's data successfully first, otherwise it can't decode its own data successfully. And the throughput of user $i$ received at user $j$ is denoted by $R_{i \rightarrow j}$, and it is given by

$$
R_{i \rightarrow j}=\log _{2}\left(1+\frac{p_{i}\left|h_{j}\right|^{2}}{n_{j}+\left|h_{j}\right|^{2} \sum_{r \in\left\{S_{n} \mid o_{r}<o_{i}\right\}} p_{r}}\right)
$$

In this optimization problem, to get the maximal sum throughput, we have to decide power allocation and decoding order in an arbitrary user group. However it is extremely complicated when we decide the two optimization variables simultaneously, so that we can decide them separately. And in some prior work [20][21], it points out that the users with higher channel gains are allocated less power and their signal will be recovered in the SIC decoding, while the users with lower channel gains are allocated much more power and their signals will be recovered by treating users' signals with less power as noise in the decoding process. Therefore it can be seen that in SIC, the optimal order of SIC decoding should be the order of increasing channel gains of users. Meanwhile when the decoding order is the order of increasing channel gains of users, the constraint $C 3$ is satisfied as constraint $C 2$ is satisfied, as when user $i$ 's data can be decoded successfully, its throughput satisfies $R_{i}=\log _{2}\left(1+\frac{p_{i}\left|h_{i}\right|^{2}}{n_{i}+\left|h_{i}\right|^{2} \sum_{r \in\left\{S_{n} \mid o_{r}<o_{i}\right\}} p_{r}}\right) \geq \widetilde{R}_{t h}$, and due to $\left|h_{i}\right|^{2}<\left|h_{j}\right|^{2}$, we have $R_{i \rightarrow j}>R_{i} \geq \widetilde{R}_{t h}$, so that we are able to remove this condition $C 3$. Hence the updated optimization problem can be formulated as

$$
\begin{array}{ll}
\max _{p} & R_{S_{n}} \\
\text { s.t. } & \mathrm{C} 1: \sum_{j \in S_{n}} p_{j} \leq P_{t}, \\
& \mathrm{C} 2: R_{j} \geq \widetilde{R}_{t h}, \forall j \in S_{n}, \\
& \mathrm{C} 3: p_{i}>p_{j}, \forall i \in S_{n} / j, o_{i}>o_{j}, \\
& \mathrm{C} 4: p_{j} \geq 0, \forall j \in S_{n} .
\end{array}
$$

\subsection{Proposed power allocation algorithm}

To tackle this problem, we consider using convex theory to acquire optimal power allocation strategy, however, it is a bit complicated to get the close-form solution. Hence, we provide insights for the optimal power allocation strategy.

THEOREM 3.1. Consider a user group $S_{n}$ with total power $P_{t}$, in the optimal power allocation strategy, user which has the best channel gain should be allocated power as much as possible in the condition that other users can be decoded successfully.

Proof: Assuming $n$ users in the user group, for notation convenience, we define $\left|h_{1}\right|^{2}>\left|h_{2}\right|^{2}>\left|h_{3}\right|^{2}>\ldots>\left|h_{n}\right|^{2}$, and we define $\Gamma_{n}=\sum_{i=1}^{n} p_{i}$, assuming a channel gain $\left|h_{x}\right|^{2}$ which satisfy the equation

$$
\begin{aligned}
& \log _{2}\left(1+\frac{\left|h_{x}\right|^{2} \Gamma_{n-1}}{\delta^{2}}\right)=\log _{2}\left(1+\frac{\left|h_{1}\right|^{2} p_{1}}{\delta^{2}}\right) \\
& +\log _{2}\left(1+\frac{\left|h_{2}\right|^{2} p_{2}}{\left|h_{2}\right|^{2} \Gamma_{1}+\delta^{2}}\right)+\ldots \\
& +\log _{2}\left(1+\frac{\left|h_{n-1}\right|^{2} p_{n-1}}{\left|h_{n-1}\right|^{2} \Gamma_{n-2}+\delta^{2}}\right)
\end{aligned}
$$


According to the order of channel gains $\left|h_{1}\right|^{2}>\left|h_{2}\right|^{2}>$ $\left|h_{3}\right|^{2}>\ldots>\left|h_{n}\right|^{2}$, we can get the relationship that

$$
\log _{2}\left(1+\frac{\left|h_{1}\right|^{2} p_{1}}{\delta^{2}}\right)>\log _{2}\left(1+\frac{\left|h_{2}\right|^{2} p_{1}}{\delta^{2}}\right)
$$

such that

$$
\begin{aligned}
& \log _{2}\left(1+\frac{\left|h_{1}\right|^{2} p_{1}}{\delta^{2}}\right)+\log _{2}\left(1+\frac{\left|h_{2}\right|^{2} p_{2}}{\left|h_{2}\right|^{2} \Gamma_{1}+\delta^{2}}\right) \\
& >\log _{2}\left(1+\frac{\left|h_{2}\right|^{2} p_{1}}{\delta^{2}}\right)+\log _{2}\left(1+\frac{\left|h_{2}\right|^{2} p_{2}}{\left|h_{2}\right|^{2} \Gamma_{1}+\delta^{2}}\right) \\
& =\log _{2}\left(1+\frac{\left|h_{2}\right|^{2} \Gamma_{2}}{\delta^{2}}\right)
\end{aligned}
$$

and as

$$
\log _{2}\left(1+\frac{\left|h_{2}\right|^{2} \Gamma_{2}}{\delta^{2}}\right)>\log _{2}\left(1+\frac{\left|h_{3}\right|^{2} \Gamma_{2}}{\delta^{2}}\right)
$$

so we have

$$
\begin{aligned}
& \log _{2}\left(1+\frac{\left|h_{1}\right|^{2} p_{1}}{\delta^{2}}\right)+\log _{2}\left(1+\frac{\left|h_{2}\right|^{2} p_{2}}{\left|h_{2}\right|^{2} \Gamma_{1}+\delta^{2}}\right)+ \\
& \log _{2}\left(\frac{\left|h_{3}\right|^{2} \Gamma_{3}+\delta^{2}}{\left|h_{3}\right|^{2} \Gamma_{2}+\delta^{2}}\right)>\log _{2}\left(\frac{\left|h_{3}\right|^{2} \Gamma_{2}+\delta^{2}}{\delta^{2}}\right)+ \\
& \log _{2}\left(\frac{\left|h_{3}\right|^{2} \Gamma_{3}+\delta^{2}}{\left|h_{3}\right|^{2} \Gamma_{2}+\delta^{2}}\right)=\log _{2}\left(1+\frac{\left|h_{3}\right|^{2} \Gamma_{3}}{\delta^{2}}\right)
\end{aligned}
$$

and the same process can be applied in next steps. At last, we can get

$$
\begin{aligned}
& \log _{2}\left(1+\frac{\left|h_{1}\right|^{2} p_{1}}{\delta^{2}}\right)+\log _{2}\left(1+\frac{\left|h_{2}\right|^{2} p_{2}}{\left|h_{2}\right|^{2} p_{1}+\delta^{2}}\right)+\ldots+ \\
& \log _{2}\left(\frac{\left|h_{n-1}\right|^{2} \Gamma_{n-1}+\delta^{2}}{\left|h_{n-1}\right|^{2} \Gamma_{n-2}+\delta^{2}}\right)>\log _{2}\left(\frac{\left|h_{n-1}\right|^{2} \Gamma_{n-1}+\delta^{2}}{\delta^{2}}\right)
\end{aligned}
$$

so there is

$$
\log _{2}\left(1+\frac{\left|h_{x}\right|^{2} \Gamma_{n-1}}{\delta^{2}}\right)>\log _{2}\left(1+\frac{\left|h_{n-1}\right|^{2} \Gamma_{n-1}}{\delta^{2}}\right)
$$

hence we can get a result that $\left|h_{x}\right|^{2}>\left|h_{n-1}\right|^{2}>\left|h_{n}\right|^{2}$ and because

$$
\begin{aligned}
& R_{S_{n}}= \\
& \log _{2}\left(1+\frac{\left|h_{x}\right|^{2} \Gamma_{n-1}}{\delta^{2}}\right)+\log _{2}\left(\frac{\left|h_{n}\right|^{2} \Gamma_{n}+\delta^{2}}{\left|h_{n}\right|^{2} \Gamma_{n-1}+\delta^{2}}\right)
\end{aligned}
$$

and power limitation $\sum_{i=1}^{n} p_{i}=P_{t}$. Assume $\sum_{i=1}^{n-1} p_{i}=$ $p_{x}$, then $R_{S_{n}}=\log _{2}\left(1+\frac{\left|h_{x}\right|^{2} p_{x}}{\delta^{2}}\right)+\log _{2}\left(1+\frac{\left|h_{n}\right|^{2}\left(P_{t}-p_{x}\right)}{\left|h_{n}\right|^{2} p_{x}+\delta^{2}}\right)$. And we can get the partial derivative

$$
\frac{\partial R_{S_{n}}}{\partial p_{x}}=\frac{1}{\ln 2}\left(\frac{\left|h_{x}\right|^{2}}{\delta^{2}+\left|h_{x}\right|^{2} p_{x}}-\frac{\left|h_{n}\right|^{2}}{\delta^{2}+\left|h_{n}\right|^{2} p_{x}}\right),
$$

because of $\left|h_{x}\right|^{2}>\left|h_{n-1}\right|^{2}>\left|h_{n}\right|^{2}$, so that $\frac{\partial R_{S_{n}}}{\partial p_{x}}>0$.

Hence, from the analysis above, we should allocate less power to user whose channel gain is the worst as long as its throughput satisfies the threshold $C 2$. And the similar proof procedure applied in the rest $n-1$ users. Therefore we can get a conclusion that user whose channel gain is the best should be allocated more power as long as rest users' signals can be decoded successfully and power allocation strategy which satisfies other constraints is shown as below.

In this part, with power constraint, we propose a power allocation algorithm, and detailed description is given in Algorithm 1. First of all, in the initial phase, users should be sorted by channel status, and power allocation process should start from the user with best channel gains, beside, their assigned power ought to satisfy the throughput. If and only if there is remaining power or total power is just enough after power allocation, current user group is feasible, otherwise, the user grouping scheme has to be updated or RRH has to assign more power to current group. Secondly, after completing power allocation initialization phase, if there is residual power, we should allocate remaining power to maximize reliable total throughput. And according to THEOREM 3.1, to maximize total throughput, residual power is prior allocated to user with best channel gain under the threshold constraint and SIC power constraint. In our proposed strategy, we combine elementary dichotomy and power allocation process. Power allocation strategy is shown in Algorithm 1.

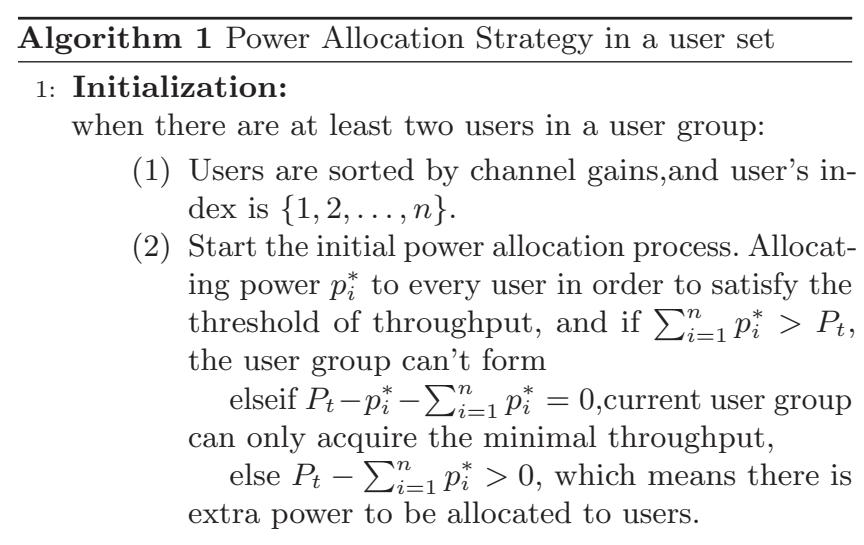

2: Extra power allocation phase:

(1) Start extra power allocating process from user whose channel gain is the best

(2) According to THEOREM 3.1, define function $\left(p_{1}\right)$.

Given the value of $p_{1}$, we can allocate power to other users according to the threshold of throughput and the constraint about power. And function $\left(p_{1}\right)=P_{t}-\sum_{i=1}^{n} p_{i}^{*}$, in which all the allocated power is obtained from Initialization. Use dichotomy to update every user's power $p_{i}^{*}$.

(3) Until function $\left(p_{1}\right) \geq \varepsilon$, in which $\varepsilon$ is a minimal value.

3: End the algorithm:

Get the result of power allocation $\mathbf{p}$.

\section{SIMULATION RESULTS}

In this section, we provide Monte Carlo simulation results in terms of sum throughput to evaluate the performance 
of proposed power allocation scheme. To get users' channel gains, we set a series of carriers and the channel amplitude gains on carriers follow i.i.d. Rayleigh distribution. Basing on the principle of NOMA, user with better channel gain is regarded to be much closer to eNB. For convenience, we randomly choose a channel in the set, and the assigned total power of user group is set as $P_{t}$, which is large enough to guarantee all users transmission in the user group in our simulations. And the AWGN for each user has unit variance (i.e., $\delta^{2}=1$ ).

In Figure 1, we compare the performance of NOMA with OMA in terms of sum-throughput and individual users' throughput when $\left|S_{n}\right|=6$, in this simulation process, the user's achievable throughput in OMA is given as $R_{i}=$ $\alpha \log _{2}\left(1+\frac{p_{i}\left|h_{i}^{2}\right|}{\alpha \delta^{2}}\right)$, and in OMA, equal bandwidth and equal transmission power are allocated to every user $(\alpha=$ $1 /\left|S_{n}\right|$ and $p_{i}=\alpha P$ ), additionally, we consider the proposed power allocation strategy as the optimal power allocation scheme in NOMA. We can see from Fig.1 that user with best channel gain will always be allocated the most power and its achievable throughput is much more in NOMA than in OMA, but contrary to other users. Besides, the NOMA system achieve more throughput than the OMA system, and all users' throughput satisfy required minimal throughput.

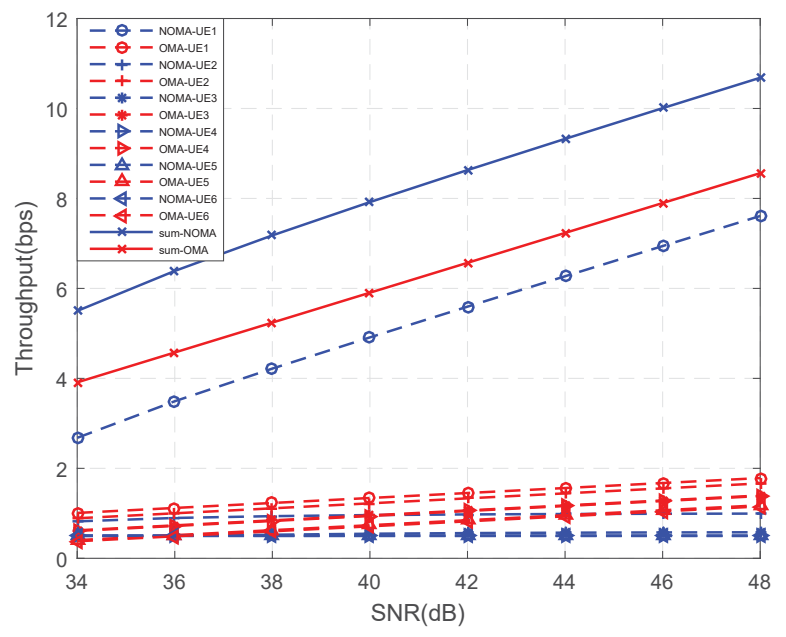

Figure 1: Throughput of 6-user downlink NOMA and OMA systems

As shown in Figure 2, the proposed power allocation scheme can achieve better performance in contrast with fractional transmit power allocation (FTPA) scheme [8] and CSI-based scheme [13]. Note that the performance difference decreases as SNR increases. And in modulation process, in the FTPA method, the transmit power of user $i$ in current user set is given as $P_{i}=\frac{P_{t}}{\sum_{j \in S_{n}}\left(\left|h_{j}\right|^{2}\right)^{-\alpha}}\left(\left|h_{i}\right|^{2}\right)^{-\alpha}$, in which $\alpha(0 \leq \alpha<1)$ is the delay factor. The case of $\alpha=0$ corresponds to the equal transmit power allocation among all users. And the larger $\alpha$ is, the more power is allocated to

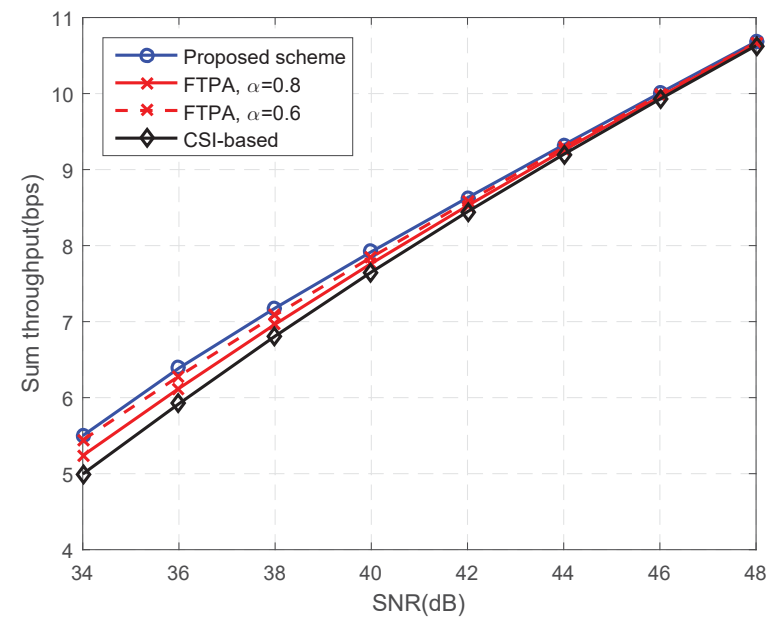

Figure 2: Throughput versus SNR for the proposed scheme and other two schcemes

the user with lower channel gain. Besides, the CSI-based method is a special case of FTPA method, in which $\alpha=1$. The assigned power of user $i$ in current user set is given as $P_{i}=\frac{P_{t}}{\left|h_{i}\right|^{2} \sum_{j \in S_{n}} \frac{1}{\left|h_{j}\right|^{2}}}$, and $\sum_{j \in S_{n}} \frac{1}{\left|h_{j}\right|^{2}}$ is the proportionality constant. From the simulation result, we can see that the sum throughput will decrease when the power allocated to user with lower channel gain increases and its performance is still better than CSI-based scheme.

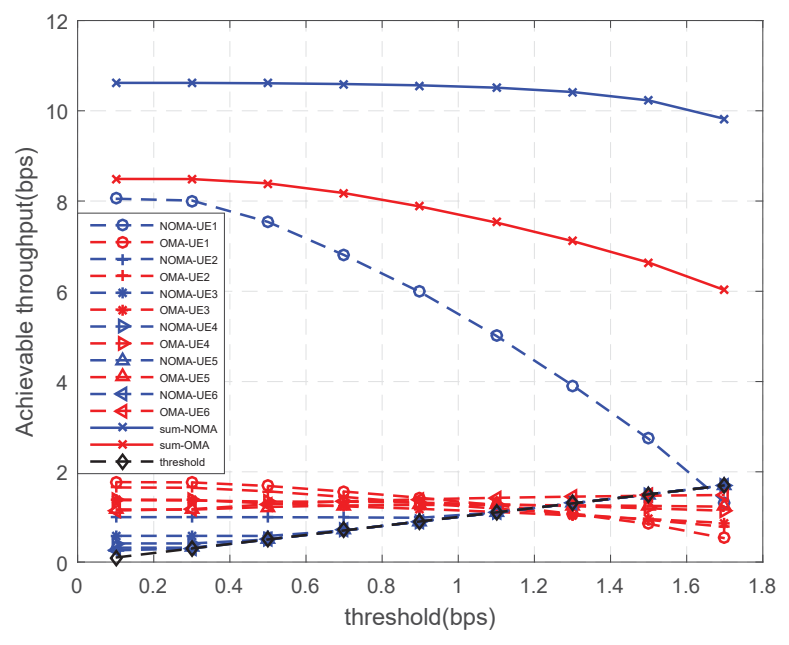

Figure 3: Achievable throughput versus different threshold

Figure 3 depicts that in a scenario with constant total transmit power, the trends of downlink sum throughput with NOMA scheme and OMA scheme is the same with increasing threshold. In both systems, achievable sum throughput decreases with increasing threshold, since much more power will be allocated to users with lower channel gains, less power 


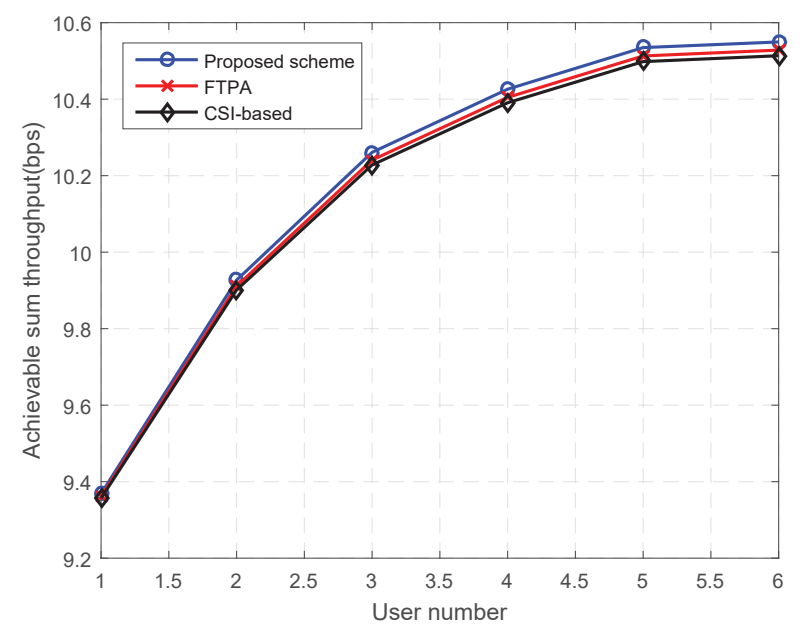

Figure 4: Throughput performances versus user number in the gruop

will be assigned to user with best channel gain and basing on THEOREM 3.1, we can know that a unit power allocated to user with better channel gain will bring more benefit. But OMA scheme performances slightly worse than NOMA scheme, owing to the fact that with increasing threshold, all users' throughput decrease. Figure 4 illustrates that the proposed scheme preforms better than the other two schemes as the number of active users increases.

\section{CONCLUSIONS}

In this paper, we have proposed a power allocation strategy for NOMA to be applied to downlink transmission in a small cell of C-RAN. According to the simulation result, it will be seen that the system performance can be improved efficiently with NOMA application. Specifically, the proposed power allocation strategy efficiently improve the achievable throughput compared with OMA system by maximizing the throughput of user with best channel gain and guaranteeing the minimum throughput of weaker user in the group. Additionally, numerical results illustrates that the proposed algorithm outperforms other schemes, under power limitation and minimum throughput requirements. It is also mentioning that current works focus on the multi-user case, and the proposed algorithm can flexibly adapt to multi-user case with much lower computational complexity. However, we only focus on a simple scenario including a small cell ignoring the constraint of fronthaul and inter-cell interference. Therefore, we are supposed to proposed optimal power allocation strategy considering a more complex scenario in further work.

\section{ACKNOWLEDGEMENT}

This work was supported in part by the State Major Science and Technology Special Projects (Grant No. 2016ZX0300 1017-004) and in part by the National Natural Science Foundation of China (Grant No. 61671074).

\section{REFERENCES}

[1] J. Andrews, S. Buzzi, W. Choi, S. Hanly, A. Lozano, A. Soong, and J. Zhang, "What will 5G be?," IEEE J. Sel. Areas Commun, vol. 32, no. 6, pp. 1065-1082, Jun. 2014.

[2] Q. Liu, H. Niu, A. Papathnassiou, and G. Wu, "5G Network Capacity: Key Elements and Technologies," IEEE Veh. Technol. Mag., vol. 9, no. 1, pp. 71-78, March. 2014.

[3] Y. Saito, Y. Kishiyama, A. Benjebbour, T. Nakamura, A. Li, and K. Higuchi, "Non-orthogonal multiple access (NOMA) for cellular future radio access," in Proc. IEEE VTC Spring, Dresden, Jun. 2013, pp. 1-5.

[4] P. Wang, J. Xiao, and L. Ping, "Comparision of orthogonal and nonorthogonal approaches to future wireless cellular systems," IEEE Veh. Technol. Mag., vol. 1, no. 3, pp. 4-11, Sep. 2006.

[5] L. Dai, B. Wang, Y. Yuan, S. Han, C.-L. I, and Z. Wang, "Nonorthogonal multiple access for 5G: Solutions, challenges, opportunities, and future research trends," IEEE Commun. Mag., vol. 53, no. 9, pp. 74-81, Sep. 2015.

[6] A. Benjebbour, Y. Saito, Y. Kishiyama, A. Li, A. Harada, and T. Nakamura, "Concept and practical consideration of non-orthogonal multiple access (NOMA) for future radio access," in Proc. Int. Symp. Intell. Signal Process. Commun. Syst., Okinawa, Japan, Nov. 2013, pp. 770-774.

[7] Z. Ding, Z. Yang, P. Fan, and H. V. Poor, "On the performance of non-orthogonal multiple access in $5 \mathrm{G}$ systems with randomly deployed users," IEEE Signal Process. Lett., vol. 21, no. 12, pp. 1501-1505, Dec. 2014.

[8] A. Benjebbour, Y. Saito, Y. Kishiyama, A. Li, A. Harada, and T. Nakamura, "System-level performances of downlink NOMA for Future LTE Enhancement," in Proc. IEEE Globcom., Dec. 2013, pp. 66-70.

[9] Y. Saito, A. Benjebbour, Y. Kishiyama, and T. Nakamura, "Systemlevel performance evaluation of downlink non-orthogonal multiple access (NOMA)," in Proc. IEEE 24th International Symposium on Personal Indoor and Mobile Radio Communications., London, United Kingdom, Sep. 2013, pp. 611-615.

[10] D. Wu, Y. Wang, H. Wang, B. Yang, C. Wang and R. Wang, "Dynamic coding control in social intermittent connectivity wireless networks," IEEE Trans. Vel. Technol., vol. 65, no. 9, pp. 76347646, Sep. 2016.

[11] D. Wu, P. Zhang, H. Wang, C. Wang, and R. Wang, "Node service ability aware packet forwarding mechanism in intermittently connected wireless networks," IEEE Trans. Wireless Commun., vol. 15 , no. 12 , pp. 8169-8181, Dec. 2016.

[12] C. Wang, J. Chen, and Y. Chen, "Power allocation for a downlink non-orthogonal multiple access system, "IEEE Wireless Communication Letters., vol. 5, no. 5, pp. 532-535, Oct. 2016.

[13] M. M. El-Sayed, A. S. Ibrahim, and M. M. Khairy, "Power allocation strategies for Non-Orthogonal Multiple Access, " in Proc. IEEE MoWNet 2016, Cario, Egypt, Apr. 2016, pp. 1-6.

[14] Z. Q. Al-Abbasi and D. K. C. So, "Power allocation for sum rate maximization in non-orthogonal multiple access system, " in Proc. IEEE PIMRC 2015, HongKong, China, Sep. 2015, pp. 1649-1653.

[15] D. P. Wu, J. He, H. G. Wang, and R. Y. Wang, "A hierarchical packet forwarding mechanism for energy harvesting wireless sensor networks," IEEE Commun. Mag., vol. 53, no. 8, pp. 92-98, Aug. 2015 .

[16] M. Peng, Y. Sun, X. Li, Z. Mao, and C. Wang, "Recent Advances in Cloud Radio Access Networks: System Architectures, Key Techniques, and Open Issues, "IEEE Commun, Surveys Tuts., vol. 18, no. 3, pp. 2282-2308, Aug. 2016.

[17] Z. Zhao, M. Peng, Z. Ding, W. Wang, and H. V. Poor, "Cluster content caching: An energy-effecient approach to improve quality of service in cloud radio access networks, "IEEE J. Sel. Areas Commun., vol. 34, no. 5, pp. 1207-1221, May. 2016.

[18] Z. Zhao, Y. Ban, D. Chen, Z. Zhao, and Y. Li, "Joint design of iterative training-based channel estimation and cluster formation in cloud-radio access networks," IEEE Access, vol. 4, pp. 9643-9658, Oct. 2016.

[19] Z. Zhao, M. Peng, Z. Ding, C. Wang, and H. V. Poor, "Cluster formation in cloud-radio access networks: Performance analysis and algorithms design," in Proc. IEEE ICC 2015, London, UK, June 2015, pp. 3903-3908.

[20] J. Liberti, S. Moshavi, and P. Zzblocky, "Successive Interference Cancellation," U.S. Patent8670418 B2, Mar. 11th. 2014.

[21] D. Tse, and P. Viswanath, "Fundamentals of wireless communication " Cambridge University Press, UK, 2015. 